Oktroi der Besatzungsmächte gegenüber dem Parlamentarischen Rat und den Ministerpräsidenten zurückkehren. Das widerspräche dem unitarischen Charakter des Bundestags“ (S.70). Im Umkehrschluss wären dann frühere deutsche Parlamente, deren Abgeordnete mit absoluter Mehrheit in Einer-Wahlkreisen gewählt wurden, Versammlungen von „Lokalrepräsentanten“ gewesen (siehe den „Unverstand im Wahlrecht“). Warum nur heißt es dann in der Verfassung des Norddeutschen Bundes und unverändert des Deutschen Reiches in Art. 29: „Die Mitglieder des Reichstags sind Vertreter des gesammten (sic!) Volkes und an Aufträge und Instruktionen nicht gebunden“? Offensichtlich wird der Charakter des Parlaments nicht von der Technik der Wahl seiner Abgeordneten abhängig gemacht. Hier rächt sich der Einstieg in die Problematik mit dem „Unverstand im Wahlrecht“, der sich als durchsichtiges Argument zugunsten des Verhältniswahlrechts entpuppt.

Politikwissenschaftlern, die sich schnell über die Judikatur des BVerfG zu Überhangmandaten informieren wollen, kann das Hauptkapitel V zur Lektüre empfohlen werden, wenn sie Meyers Standpunkt, den sie nicht teilen müssen, dabei berücksichtigen. Für an Wahlsystemen allgemein interessierte Politikwissenschaftler sind andere Kapitel eher obiter dicta.

Franz Urban Pappi

\title{
Bundespolitik und Landtagswahlen: Alltagsverständnis untermauert durch wissenschaftliche Analyse
}

Völkl, Kerstin, Kai-Uwe Schnapp, Everhard Holtmann und Oscar W. Gabriel: Wähler und Landtagswahlen in der Bundesrepublik Deutschland (Studien zur Wahl- und Einstellungsforschung, Band 10), Nomos Verlagsgesellschaft, Baden-Baden 2008, 489 Seiten, € 59,-.

Sieben Landtagswahlen in Hamburg, Sachsen-Anhalt, Baden-Württemberg, RheinlandPfalz, Bremen, Mecklenburg-Vorpommern und Berlin sowie auch noch in Nordrhein-Westfalen bestimmten 2011 das (partei-)politische Geschehen. Ohne Berücksichtigung der landesweiten oder regionalen Kommunalwahlen in allen Bundesländern könnte diese Studie zur Wahl- und Einstellungsforschung - wie schon die Dissertation der Mitherausgeberin Kerstin Völkl von 2009 - aktuell die Auswirkungen der Bundespolitik auf die Landtagswahlen in einem Drittel, vielleicht der Hälfte der Bevölkerung untersuchen. Für die Politik ist die Antwort eindeutig: Baden-Württemberg stimmte über die schwarz-gelbe Bundesregierung und Deutschlands wirtschaftlich-technologisches Schicksal ab; Bremen als kleinstes Bundesland lief beim erwartbar klaren Ergebnis unter ferner liefen. Die Landtagswahlen in Niedersachsen und Bayern 2013 gelten für alle Politiker als klarer Hinweis auf die anschließende Bundestagswahl.

Für die Autoren ist die Lage nicht so klar. Uni sono trennen sie, auch aufgrund von US-Studien, die Wahlentscheidung jedenfalls der meisten Wähler für den Bundestag von

1 Kerstin Völkl, Reine Landtagswahlen oder regionale Bundestagswahlen? Eine Untersuchung des Abstimmungsverhaltens bei Landtagswahlen 1990-2006, Baden-Baden 2009; vgl. zu diesem Buch Ossip Fürnberg, Wahlverhalten bei Landtagswahlen: Gute Basis für weitere Forschung, in: ZParl, 41. Jg (2010), H. 4, S. 927 f.; kritisch Erich Röper, in: VR 2010, S. 359. 
jener für ihr Landesparlament. Die Herausgeber erläutern den theoretischen Rahmen und die Analysemodelle zur Wirkung der Bundespolitik auf Landtagswahlen. Im Großen und Ganzen nach einheitlichem Muster präsentieren die 16 Länderbeschreibungen die Einzelheiten: Der Einleitung folgen die immer gleiche Beschreibung des theoretischen Rahmens mit Aggregat- und Individualebene sowie die Datenlage; die im wesentlichen einheitliche Methode zur empirischen Analyse mit den Bundes- und Landtagswahlergebnissen der vergangenen Jahrzehnte sowie ein Fazit; danach Literaturangaben und ein Anhang über die Umfrageergebnisse. Auch die Ergebnisse sind im Grundsatz gleich. Zwar nennen einige Autoren bundespolitische Einflüsse, sie kehren aber bald zurück zum Mainstream. Bei aller Differenzierung geht es um weitgehend eigenständiges Landtagswahlverhalten. Doch bestätigt sich auch für politische Praktiker „die Feststellung von [Reiner] Dinkel , dass sich die ,Ergebnisse von Landtagswahlen als Addition einer systematischen Beeinflussung durch die Konstellation im Bundestag und der politischen Situation im jeweiligen Land' bilden“" (S. 283).

Die Wirkung der Bundespolitik auf Landtagswahlen hängt unter anderem ab von Struktur und Größe des Bundeslands und auch einer Art Landesbewusstsein. Daher macht es wenig Sinn, in gleicher Weise Bremen und Nordrhein-Westfalen ${ }^{3}$ zu betrachten. Im ersten Fall sind es - schon wegen der getrennten Wahlbereiche Bremen und Bremerhaven - eher „aufgenordete“ Kommunalwahlen. ${ }^{4}$ An Rhein und Ruhr spricht „für die These der Bundestestwahlen und einer starken bundespolitischen Durchdringung der Landtagswahlen unser Befund, demzufolge es für jede der von uns untersuchten Landtagswahlen in NordrheinWestfalen einen starken und signifikanten Einfluss der Bundesebene gibt. Die Bewertung der beiden großen Parteien auf der Bundesebene erweist sich bei jeder Landtagswahl als eine wichtige Bestimmungsgröße der Wahlentscheidung" (S. 235). Gerhard Schröder und Franz Müntefering begriffen es 2005 ebenso wie Angela Merkel 2009. Aber da es wohl dem Forschungsvorhaben widerspricht, heißt es wenig später: „Wenn wir im Vergleich ermitteln, welchen Effekt die Bewertungen der Bundesparteien und die Bewertungen der Landesparteien auf die Landtagswahlentscheidungen ausüben, so zeigt sich für alle drei Landtagswahlen seit 1985 ein konsistent stärkerer Effekt der Bewertungen der Landesparteien. “ Die Wirkung von Hartz IV auf das Entstehen der WASG, die Fusion mit der PDS zur Linken und ihr Erfolg bei der Bundestagswahl 2009 sowie den Landtagswahlen auch im Westen sprechen gegen die Vorgaben der Studie.

Kritisch ist anzumerken, dass bei der Wahlbeteiligung nur auf die Prozentzahlen, nicht auf absolute Zahlen rekurriert wird. So bleibt etwa offen, ob die Wahlbeteiligung von nur

2 Reiner Dinkel, Der Zusammenhang zwischen Bundes- und Landtagswahlergebnissen, in: PVS, 18. Jg. (1977), H. 2, S. $348-359$.

3 So sahen sich vor der Landtagswahl 1985 nur 2,3 Prozent der Bewohner als Nordrhein-Westfälinger.

4 Typische Ungenauigkeiten fallen hier sehr auf: Bis 1949 waren NS-Belastete nicht wahlberechtigt, was dann bis 1961 zum großen Erfolgt der DP, SRP und DRP und Schwäche der CDU führte. Eine große Koalition gab es 1951/59 und 1995/2007; auch 2003 war Henning Scherf SPD-Spitzenkandidat und Bürgermeister. Die bundespolitische Bedeutung der SPD zeigt, dass Hans Koschnick 1975/79 stellvertretender Bundesvorsitzender war. Mit dem Erfolg der Bremer Grünen Liste als auch personeller Vorläufer der Grünen hätte sich der Autor mehr befassen müssen. Den Niedergang der Bremer Stahlindustrie gibt es nicht. Die Bürgerschaft ist die Bremische und der Schütting ist die Handelskammer, nicht das Gildehaus der Handwerkskammer. 
44,4 Prozent in Sachsen-Anhalt am 26. März 2006 nicht in Wahrheit ein auf die Bundesebene (Hartz IV) zielender Protest war. Für jemanden, der viele Wahlen mitplante, administrierte und beobachtete, wären Erkenntnisse statt des „sowohl als auch“ hilfreich, wenn es etwa für Bayerns Wähler heißt: „Wenn sie ihre Landtagsstimme abgeben, sind [sie] für Bundeseinflüsse zugänglich, lassen sich aber beileibe nicht nur von diesen beeinflussen“" (S. 87). Sollen nun im Wahlkampf Bundespolitiker herangezogen oder auf sie verzichtet werden, wie etwa im Fall Guido Westerwelles, der in Rheinland-Pfalz nicht auftreten sollte? Und welcher Ebene sind Bundespolitiker wie Gerhard Stoltenberg 1971 oder Olaf Scholz 2011 als Spitzenpolitiker bei Landtagswahlen zuzuordnen?

Sachgerecht ist der eigene Abschnitt für die östlichen Bundesländer. Noch besser wäre er gelungen, wenn die Ausgangslage und die Folgen der Einheit einbezogen worden wären: Statt blühender Landschaften flächendeckend Deindustrialisierung, westdeutsche Glücksritter und „Berater“ (oft mit „Buschzulage“), massenhaft Arbeitslosigkeit und Abwicklung in Behörden und öffentlichen Unternehmen, Westdeutsche in fast allen Spitzenpositionen auch der Wirtschaft, andererseits die Stärke der PDS/Linken; die Liste ließe sich fortsetzen. ${ }^{5}$ Daher ist es in der Tat wenig sinnvoll, diese Bundesländer nach dem westlichen Schema zu bewerten, zu fluid sind gesellschaftspolitische Struktur und Parteien. So sei für Thüringen die Frage nach bundespolitischen Einflüssen auf die Landtagswahlergebnisse nicht abschließend zu beantworten (S. 426); und Mecklenburg-Vorpommerns CDU sieht auf das Wahlergebnis in Sachsen-Anhalt, nicht in Baden-Württemberg.

Auch wäre es besser gewesen, Bremen, Hamburg und Berlin (getrennt nach West und Ost) nicht im Kontext der Flächenländer zu behandeln. Zu sehr haben dort, wo jeder jeden Handelnden kennt und mit Ortsgespräch erreichen kann, örtliche Einflüsse große Bedeutung. So „zerlegen“ sich in Bremen CDU, FDP und Linke, gibt es Wählerinitiativen, fehlt in Hamburg ein überzeugender CDU-Spitzenkandidat, leidet Berlins CDU noch unter dem Bankenskandal, ist die Stadt gespalten, wie die Volksabstimmung zur sachfremden Vereinigung mit Brandenburg (Art. 118a GG) zeigte. Wie ist Olaf Scholz' Erfolg erklärlich, wenn „bei den Hamburger Bürgerschaftswahlen [Wahlerfolge von Parteien, die auch in Berlin regieren] wahrscheinlicher als der umgekehrte Fall sind“ (S. 170)?

Hilfreich schiene es auch, die Flächenländer nach der Bevölkerungszahl zu sortieren. In den großen sind „kleine Bundestagswahlen“ wie 2005, 2010 und 2012 in Nordrhein-Westfalen oder jüngst in Baden-Württemberg wahrscheinlicher - zumal es dort kein ausgeprägtes Landesbewusstsein gibt - als im Saarland, wenn es nicht gerade um den Bundespolitiker Oskar Lafontaine geht. Als apercu: 53,1 Prozent für die CDU am 24. März 1974 in Schleswig-Holsteins Kreisen und kreisfreien Städten nach rund 45 Prozent zuvor und unter 50 Prozent danach sind nur erklärlich durch die sich verdichtende Guillaume-Affäre und die Schwäche Willy Brandts.

So mag man überspitzt resümieren: Wenn der Hahn kräht auf dem Mist, ändert sich das Wetter, oder es bleibt wie es ist. Nur: Plausibles Alltagsverständnis mag oft hilfreich sein; es gewinnt aber an Kraft, wenn es durch wissenschaftliche Analyse substantiiert wird.

Erich Röper

5 Dazu Erich Röper, Die minderen Brüder und Schwestern, in: APuZ B 40 (2005), S. 19 - 25; ders., Allzeit verschiedene Deutsche, in: KritV 2001, S. 5 - 23. 